\title{
Safe Beacon: A Bluetooth Based Solution to Monitor Egress of Dementia Sufferers within a Residential Setting ${ }^{\dagger}$
}

\author{
Joseph Rafferty 1,* , Jonathan Synnott ${ }^{1}$, Chris Nugent ${ }^{1}$, Ian Cleland ${ }^{1}$, Andrew Ennis ${ }^{1}$, \\ Philip Catherwood ${ }^{1}$, Claire Orr ${ }^{1}$, Andrea Selby ${ }^{2}$, Gary McDonald ${ }^{3}$ and Gareth Morrison 4 \\ 1 Faculty of Computing, Engineering and the Built Environment, University of Ulster, \\ Jordanstown BT37 0QB, UK; j.synnott@ulster.ac.uk (J.S.); cd.nugent@ulster.ac.uk (C.N.); \\ i.cleland@ulster.ac.uk (I.C.); a.ennis@ulster.ac.uk (A.E.); p.catherwood@ulster.ac.uk (P.C.); \\ c.orr@ulster.ac.uk (C.O.) \\ 2 Kirk House Care Home, Belfast BT5 7BX, UK; aselby@belfastcentralmission.org \\ 3 Limitless Insight, Belfast BT3 9DT, UK; gary@limitlessdigitalsoutions.com \\ 4 The Lava Group, Belfast BT12 6RD, UK; g.morrison@thelavagroup.co.uk \\ * Correspondence: j.rafferty@ulster.ac.uk \\ + Presented at the 12th International Conference on Ubiquitous Computing and Ambient Intelligence \\ (UCAmI 2018), Punta Cana, Dominican Republic, 4-7 December 2018.
}

Published: 22 October 2018

\begin{abstract}
The global population is ageing, as a consequence of this there will be a greater incidence of ageing related illnesses which cause cognitive impairment-such as Alzheimer's disease. Within residential care homes, such cognitive impairment can lead to wandering of individuals beyond the boundaries of safety provided. This wandering, particularly in urban areas can be life threatening. This study introduces a novel solution to detect, and alert caregivers of, egress of at-risk inhabitants of a care home. This solution operates through a combination of wearable Bluetooth beacons and beam-formed listening devices. In an evaluation process involving 275 egress events, this solution proved to offer accurate operation with no incidence of false positives. Notably, this solution has been deployed within a real residential care home environment for over 12 months. Proposed future work discusses improvements to this solution.
\end{abstract}

Keywords: Ambient Assistive Living; dementia; egress detection; smart home; smart environments; ageing care; bluetooth beacons

\section{Introduction}

The composition of the global population is changing in an unpreceded manner. Specifically, the median age of the global population is rising wherein that median age is predicted to increase from 28 in 2009 to 38 in 2050 [1,2]. This phenomenon has been called global aging.

Several factors have contributed to this global aging, including reduced levels of adult mortality and a decrease in birth rates [2-5]. In regions which experienced the post-World War II baby boom, such as Europe and the United States of America, this aging is further compounded [3-5].

Evidence based prediction indicates that this global aging will lead to a demographic where by 2050 over $20 \%$ of the population will be the age of 65 or over $[1,2,6]$. Such a demographic transition will invariably cause an increase in aging related illnesses, such as Dementia. An increase in these illnesses will therefore increase the care requirements for the aging portion of the population.

Ambient Assistive Living (AAL) is an approach to augment care for individuals through technological means. AAL solutions may be integrated into an individual's environment and 
combined with other non-environmental components such as services and applications. AAL has been seen as a promising approach to reduce the overhead of care to individuals while simultaneously.

Alzheimer's disease is an ageing related illness which has several symptoms which cause issues for individuals suffering from it and those caring for them [5,7-11]. These issues include:

- Challenges with planning or problem solving, such as inability to plan appropriate routes

- Changes in mood and personality, such as an increase in anxiety/fear

- Disruptive memory loss, such as misplacing items or relying on memory aids

- Issues understanding visual images, such as warning signs or road crossing lights

- Issues comprehending spatial relationships, such as distances between roads and pavements

- Impairments to judgement, such as crossing a road when traffic is rapidly approaching

- Withdrawal from social or work activities/settings, such as wanting to leave social gatherings

These issues can contribute to a number of troublesome/dangerous behaviors such as wandering, mismanaging money, angry outbursts, sleep issues and unsafe/unmonitored egress from a residential environment [5,7-11]. The combination of these behaviors and symptoms can introduce a potential risk to life. This risk is especially pronounced in the scenario where unsafe/unmonitored egress from a residential environment occurs at night by a confused individual within an urban setting.

Previously, AAL has be used to help alert caregivers when troublesome behaviors such as wandering or unsafe egress occur [9,12-14]. As such this study aimed to devise, evaluate and deploy a solution that identifies unsafe egress from a residential environment. To facilitate this a custom solution was devised and developed which was based around wearable Bluetooth beacons which operate in conjunction with a listening device to provide reliable detection of presence in a location that indicates egress.

Related work is presented in Section 2, the devised solution is detailed in Section 3, Section 4 presents an evaluation of the platform and Section 5 concludes offering insight into future work.

\section{Related Work}

A plethora of solutions to detect egress and location of individuals exist, however, these have some deficiencies making them unsuitable for use given the restrictions of the intended deployment scenario.

Helmy et al. [14] have produced an app based solution to monitor the location of persons with Alzheimer's disease and Autism Spectrum Disorder. This requires a person to be monitored to carry a smart phone on their person which contains an application which uses GPS to determine their location. This location data is then relayed to a processing service. This location is subsequently compared against caregiver specified "zones" to determine if an at-risk individual is in dangerous area, such as the outside of a care home. In the event that a person is in a dangerous area the supporting/caregiving personnel are alerted. This solution has shown promise; however, it has a number of deficits which make it incompatible with the requirements. Notably, it has a reliance on external GPS signals, has a short battery life and relies on the device to be carried by an at-risk individual to have an internet connection.

Cantón Paterna et al. [15] devised a solution to locate individuals within an environment. This solution accurately locates Bluetooth LE based devices, such as those placed on an at-risk individual, within an environment using multiple Bluetooth listeners. These Bluetooth listeners monitor the received signal strength from observed Bluetooth LE devices. The signal strength data from these listeners is processed by a trilateration algorithm which is augmented with Kalman filters. The output of this processing is highly accurate location of Bluetooth LE devices. This solution could be adapted to determine location of an individual and generation of alerts of when they enter an unsafe zone. This solution has deficiencies for the desired use case related to the quantity of listeners and the complex modelling required for operation. Although this could potentially be used to detect wandering of Alzheimer's suffers, it was not evaluated to be used in this capacity. 
Lin et al. [16] proposed a solution to track individuals using Bluetooth LE based beacons. In this solution persons of interest were issued with Bluetooth LE based tags, typically to be integrated into their clothing or personal affects. Whenever their wandering from a residential environment is noticed by caregivers, the unique identifier for the Bluetooth LE beacon is published to a crowd sourced network of applications. Each application within this network is deployed to smart devices, such as phones and tablets, owned by volunteers. Each application instance in that network scans their vicinity for the identifiers of Bluetooth LE beacons that have reported as missing. If an application instance observes such a beacon, then the location of that smart device is determined though GPS and relayed to a central service. Subsequently, this central service will relay the location to concerned parties such as caregivers and family members. This solution has a number of deficiencies. Notably it relies on caregivers to notice the absence of an individual and publish their Bluetooth beacon identifier. Also, it relies upon a crowd-based installation to exist and have brow members within the vicinity of a missing individual. A comparable solution has been produced by Issoufaly et al. [17].

Radio Frequency IDentification (RFID) based tracking solutions, such as [18,19], may be adapted to track users location to determine their presence in regards to unsafe zones. These solutions leverage trilateration of RFID devices through multiple listener stations. Passive RFID tags have an advantage where they do not require a local power source. However, much like other trilaterationbased approaches, this solution requires extensive infrastructure and complex modelling required for reliable operation.

Computer vision based approaches such as those in $[20,21]$ may be used to identify the presence of individuals of interest within designated areas, such as the exit of a residential environment. Such solutions rely upon detection of discriminative features of individuals, typically though facial recognition or gait analysis [20,22-26]. However, the accuracy of such discriminative approaches is currently insufficient to accurately identify specific individuals reliably, this is especially notable when observing video cameras are not viewing subjects at optimal angles. Additionally, these solutions have issues with variable lighting conditions and headwear- as would occur in the natural world. Finally, such computer vision-based approaches are computationally intensive-reducing their scalability.

In summary, deficiencies of current solutions include [27,28]:

- A reliance on satellite/GPS based geofencing to function, the signals powering such satellite based solutions may not always be received in a timely manner [14,29-31]

- A Requirement for monitored persons to carry devices with a relatively short battery life (i.e., under 1 week) [14]

- A Reliance on trilateration-based operation which requires a relatively complex infrastructure and customization of advanced algorithms to function reliability $[15,27]$

- A necessity for the monitored persons to carry a device which can maintain a constant internet connection

- A reliance on caregivers to notice the absence of an at-risk individual

- Dependence on a crowdsourced network of application installations to exist

Notably, this study intended to deploy this solution within a residential care home and had several requirements, which were:

- Reliable operation without incorporation of potentially reliable satellite/GPS signals

- Operation though a reduced infrastructure, the environment would not support deployment of sufficient listening stations to support trilateration

- The solution would only need to be used when at risk individuals are a tenant of the general residential environment which has access to the external environment, a removable/on-demand, end user deployable, solution would be more suited to this use

- Tracking of egress through simple wearable artefacts which provide a lengthy battery life to reduce maintenance, ideally over three months 
In order to address these deficiencies, a custom solution was devised and evaluated. This solution is detailed in Section 3 and results of this evaluation are presented in Section 4.

\section{Safe Beacon: A Bluetooth Based Solution to Monitor Egress of Dementia Sufferers within a Residential Setting}

In order to address these deficiencies, a custom solution was devised-called Safe Beacon. Central to this egress monitoring solution are a number of requirements, as previously outlined in Section 1. Within the design of this solution, inhabitants of the smart home are supplied with wearable Bluetooth LE beacons. Bluetooth beacons are devices which broadcast data such as temperature, acceleration data, a URL or simply their presence. These broadcasts incorporate a unique identifier for each individual beacon.

These beacons are monitored by Bluetooth listening units logically placed at points of entry/exit.

The listeners monitor the MAC address of Bluetooth LE beacons within their vicinity and relay the associated Received Signal Strength Indication (RSSI) to a server backend. This RSSI can be used to roughly indicate distance from a listener [32]. However, detection is omnidirectional, as dictated by the common design goals of the antenna involved. As such, these listening units incorporate Electromagnetic shielding to attenuate the signal received by their antennae [33]. This enables a significant reduction in RSSI from specific approach vectors, facilitating beamforming thus reducing false positives where an inhabitant loiters near the listeners but has not exited the building.

These RSSI measurements are relayed to a cloud based architecture which incorporates the SensorCentral platform [34]. These per beacon RSSI values are then processed by an alerting daemon which will issue an alert when a threshold RSSI value has been reached.

Alerts are relayed using the SensorCentral SDK via Firebase Cloud Messaging [35] to an alert management application. This alert management application is installed on devices that on duty caregivers have on their person, enabling them to respond to the potentially dangerous situation which is unfolding, in this specific use scenario this is egress of a person with dementia to a busy, and congested, urban environment.

Section 3.1 provides some background to the principles of Bluetooth based egress detection employed. Section 3.2 presents detail on the overall architecture of the solution. Section 3.3 elaborates on the low-cost commodity devices that are used to realize the solution and the rationale behind their selection. Section 3.4 provides information on the alert management application.

\subsection{Bluetooth Based Egress Detection}

Using Bluetooth based listeners it is possible to determine inferred distance of Bluetooth based transmission devices, such as wearable Bluetooth Beacons. This inferred distance is determined by the RSSI, the strength of a detected signal expressed in decibel-milliwatts $(\mathrm{dBm})$. The higher the $\mathrm{dBm}$ value the closer the transmitting device is to a listener. The RSSI scale for Bluetooth devices is between $0 \mathrm{dBm}$ and $-127 \mathrm{dBm}[32,36]$. A RSSI of $-126 \mathrm{dBm}$ indicates that a beacon is at the limits of detection for a specific listener. Conversely, a RSSI value of $0 \mathrm{dBm}$ indicates that the device is likely as close to the listener as possible.

Leveraging this ability to detect proximity, it is possible to use trilateration to detect the location of an individual. Such a trilateration-based approach relies upon careful and considered placement of several of listeners. These placement parameters subsequently need to be incorporated into the trilateration algorithm. This represents a financial and logistical overhead to deployment of such solutions. However, using a single listener in combination with Electromagnetic Shielding it is possible to detect egress using a single listener. This is possible through attenuation of RSSI values to create a beamformed detection zone. In essence, a zone is created through shielding to reduce observability outside the desired zone. This is illustrated with the aid of Figure 1. Figure 1a depicts RSSI values from beacons $A$ and $B$ as detected by a listener without shielding. Figure $1 \mathrm{~b}$ depicts RSSI values from beacons A and B when shielding is in place between Beacon B and the listener. 


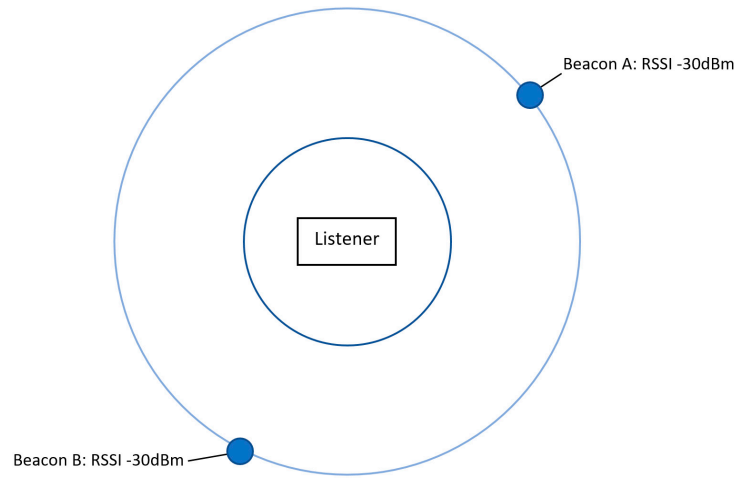

(a)

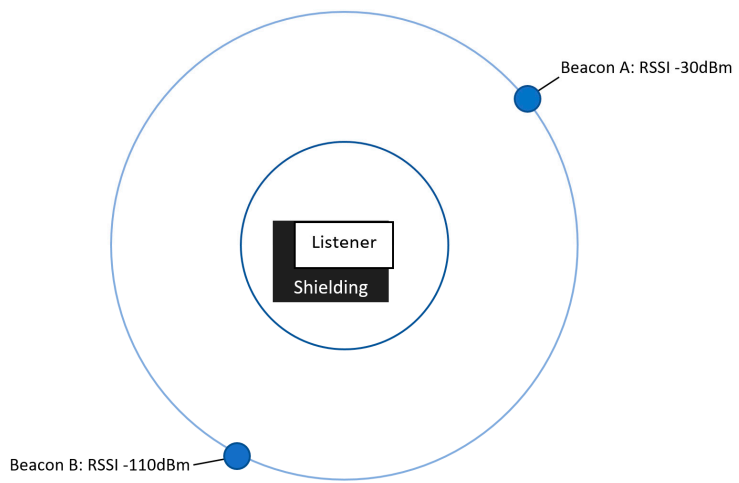

(b)

Figure 1. The use of shielding to create a beamformed egress detection zone: (a) presents RSSI values detected from two beacons where there is no shielding in place-they are both equal; (b) presents RSSI values from two beacons where shielding is in place between a listener and Beacon B.

An early proof of concept listener housing to confirm this effect and its suitability to produce a beamformed egress detection zone is presented in Figure 2.

This proof of concept indicated that even with modest shielding, significant attenuation of RSSI signals can be achieved. This proof of concept design was then revised with better shielding and a more capable listener-this is discussed later in the body of this manuscript.

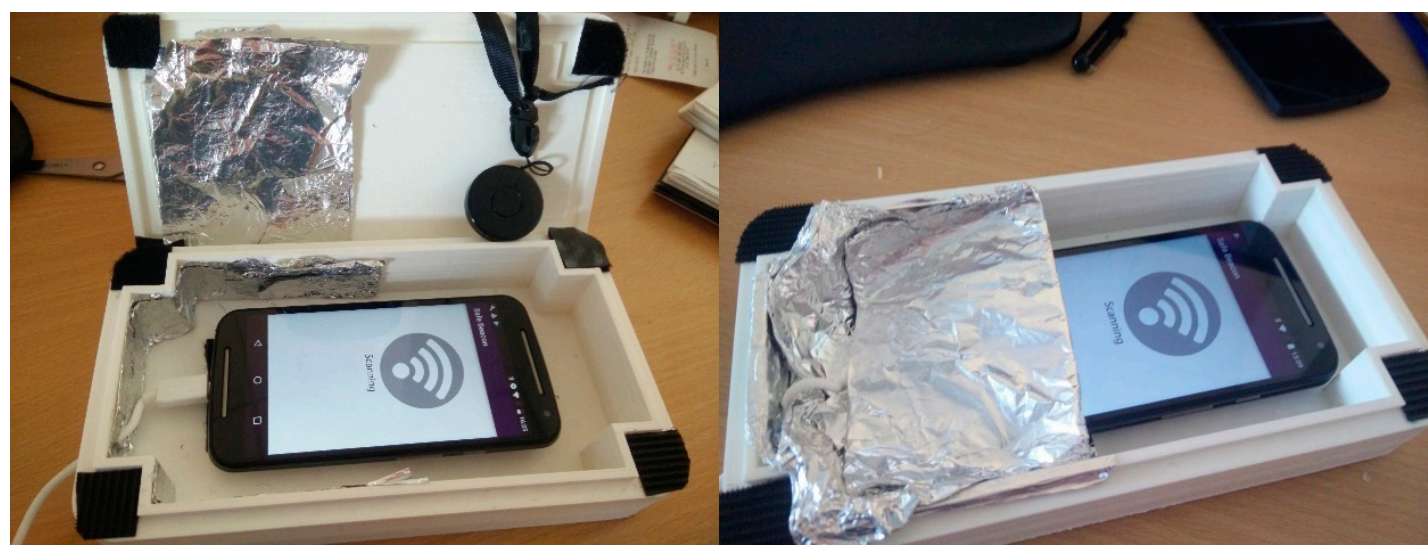

Figure 2. A proof of concept listener to evaluate the viability of electromagnetic shielding to provide a beamformed egress detection zone.

\subsection{Architecture}

This solution leverages the SensorCentral platform to manage, store and query sensor data from the listeners. Listeners provide this sensor data by scanning for local Bluetooth beacons at a rate of 10 $\mathrm{hz}$ and then relays this data to a backend service via REpresentational State Transfer (REST) [37].

This sensor data consists of three core parameters Beacon ID, RSSI and sensor listener ID. Beacon ID address is a globally unique address for detected Bluetooth devices, derived from a unique hardware address. RSSI is expressed as signed integer representing dBm values. The sensor listener ID indicates the listener which detected the beacon. In addition to storing this core data, SensorCentral stores metadata related to the Bluetooth listeners and Beacons.

In regard to listeners, metadata stored includes location, friendly label, and sensor listener ID. The location is a label such as "Kirk House Care Home-Front Door". The friendly label may be shorter and more relatable such as "Front door", this enables incorporation into more relatable alert messages. Sensor listener ID is stored, such as 353310060701766_9_69, this in the default SensorCentral UUID format as explained in [34]. Metadata stored related to beacons includes a Beacon ID, friendly label and location. Use of a Beacon ID reduces ambiguity and enables processing of per user data. The friendly label is used to identify the associated user, such as "Sandy Clemand". The location 
metadata is updated on detection of an egress event-indicating the listener that has detected the egress.

This related sensor data is processed by an alert daemon which will issue an alert when a RSSI reaches a threshold value indicating an egress event has occurred. When an alert is generated, the Beacon ID is used to determine its friendly label, such as "Sandy Clemand", which becomes the subject of the alert. The sensor listener ID is then used to determine its location as registered in its friendly label. This enables subject and location to be combined into an alert such as "Sandy Clemand detected @ Front Door". This alert message is subsequently forwarded to the alert management application via Firebase Cloud Messaging. The overall architecture of the solution is presented in Figure 3.

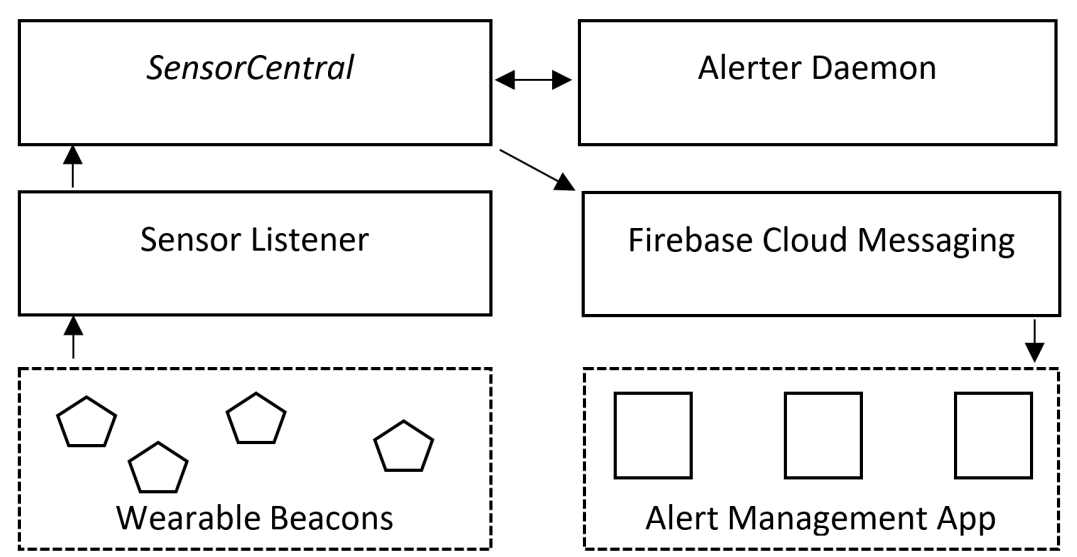

Figure 3. The general architecture of the devised solution, as described within this section.

Central to this architecture is the SensorCentral big data platform, which stores and manages data relayed from the sensor listeners in a storage engine consisting of a time series database and a document-oriented database. The architecture of this platform is presented in Figure 4, this is reproduced from [38] with permission.

The devised solution relies upon number of hardware components integrated into the architecture presented in this Section. These hardware components are enumerated in Section 3.3.

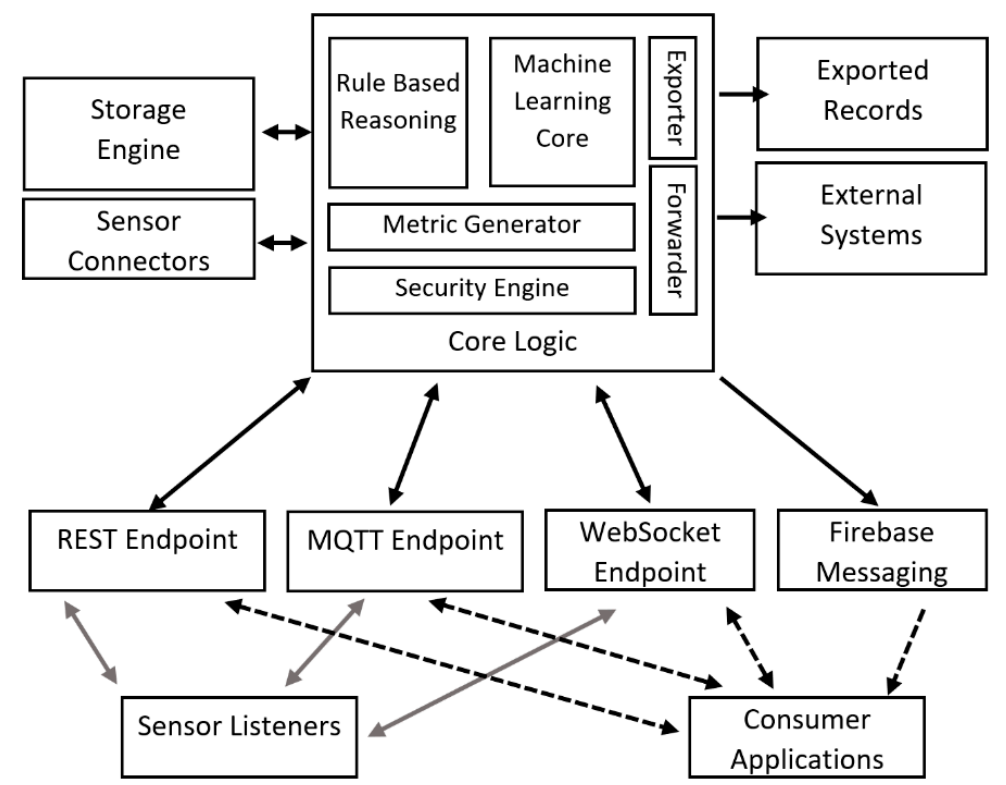

Figure 4. The general architecture of the SensorCentral component of the architecture. This is this is reproduced from [38] with permission. 


\subsection{Hardware Components}

The hardware components of this solution are broadly in the categories of listeners and wearable beacons. Beacons broadcast their unique Bluetooth identity in conjunction with other data such as a heartbeat packet or data representing temperature readings. In this scenario beacons should ideally only send heartbeat packets due to energy use considerations. However, beacons which rely more than heartbeat packets at the expense of energy consumption will be compatible with the devised solution. In this instance, the Bluetooth beacons involved only need to relay heartbeat information, with inferred RSSI parameters. In this solution two types of wearable beacons were chosen, these are lanyard mounted beacons and wrist worn beacons. Both of these beacons have an advertised battery life of over 6 months and are presented in Figure 5.

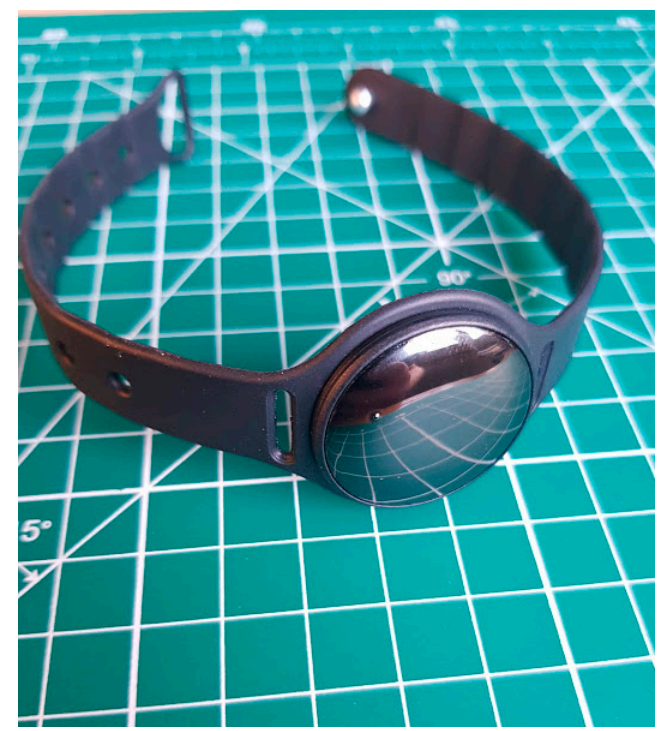

(a)

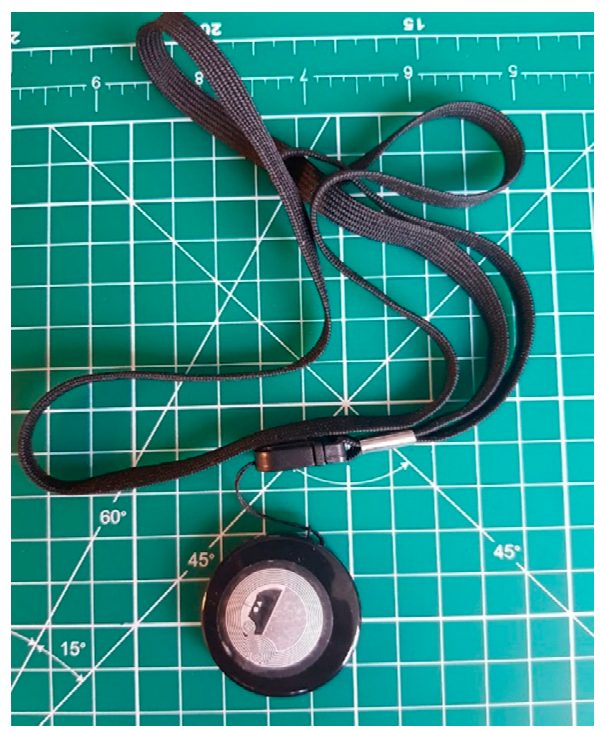

(b)

Figure 5. The wearable Bluetooth beacons that were incorporated into this solution: (a) depicts the wrist worn beacon type that was adopted; (b) depicts the adopted lanyard worn beacon type.

In addition to wearable Bluetooth beacons, Bluetooth listeners are required. During development of this solution, three listening components were evaluated with two of these accepted as viable components to the platform.

Two of these evaluated devices were Android based smartphones that ran a custom listening application and one was a dedicated single board computer which operated a Python-based listening daemon. The Android smartphones were a Moto G and Nexus 5. The single board computer was a Raspberry Pi 3 model B. These listeners were evaluated to determine performance, as presented in Section 4. This evaluation revealed that the Nexus 5 and Raspberry Pi were the only suitable listener platforms for the solution, out of those that were tested. The Moto $G$ encountered issues related to a reduction of its polling rate in the listener process when it operated for a long time. As such, did not reliably detect egress simulations, this was attributed to thermal throttling on its CPU.

The data from these listener components are stored in SensorCentral and processed by the alerter daemon as presented in Sections 3.1 and 3.2. When an urgent condition is detected this is relayed to instances of Alert Manager applications as presented in Section 3.4.

\subsection{Alert Management}

When the alerter daemon determines that an individual has exited the residential environment, it will $\log$ an alert within a document-database and issue a notification to installations of the alert management application. This alert management application receives these notifications via the Firebase Cloud Messaging service. On receipt of this notification, the application will alert caregivers with an audible alarm and pop up message. On clicking these alerts, or opening the application, 
caregivers can view the alert text, time and location. Alert text is similar to "Sandy Clemand detected@Front Door", as detailed previously. This alert management application is presented in Figure 6.

This alert management application was developed using the Ionic framework [39]. This framework enables production of cross platform applications with a shared code base using the Angular framework [39]. As such, this solution enables caregivers to install the application on any device which runs Android, iOS, Blackberry and Windows 10 platforms.

This solution was evaluated extensively during: development, lab-based evaluation and within a real environment, this evaluation is presented in Section 4.

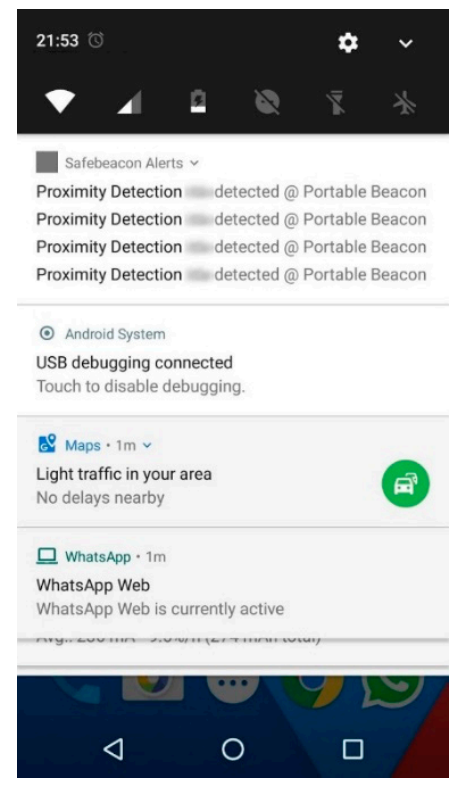

(a)

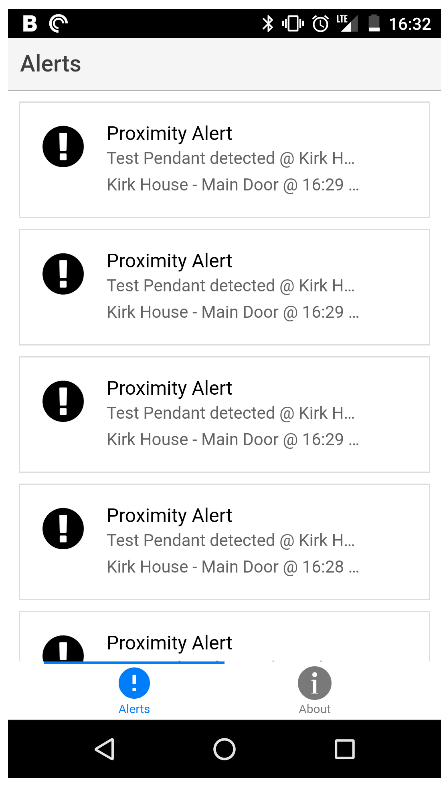

(b)

Figure 6. The alert management application that is integrated into this solution: (a) depicts alert notifications received via push messaging; (b) depicts a record of alerts generated by the system.

\section{Evaluation}

This solution was evaluated at three stages. Stage 1 was an evaluation of the capability of three candidate listener devices, this is presented in Section 4.1. Stage 2 was a lab-based evaluation of the solution with listener devices, this is presented in Section 4.2. Stage 3 was an evaluation of the solution within a caregiving residential environment performed independently by caregivers, this is presented in Section 4.3 .

\subsection{Evaluation of Candidate Listener Devices}

These listeners were two Android based smartphones and a single board computer. The Android phones were a Moto G and Nexus 5. The single board computer was a Raspberry Pi 3 model $B$. The objective of this evaluation process was to determine reliable operation over a long term.

The evaluation occurred over several weeks where each listener was operated in a workspace and periodic testing occurred. The testing process involved moving a Bluetooth LE beacon into the detection zone for that listener, if the listener detected the beacon an alert was issued.

Initial testing occurred with a Moto $G$ phone acting as a listener. During this evaluation it initially appeared that the Moto $G$ accurately detected the presence of Beacons within the alert zone. However, following long term operation of the device detections would not occur unless the beacon loitered for an unreasonable amount of time. After some further testing it was determined that thermal throttling of the smart phone was the root cause of this issue.

Following this, testing with a better specified smartphone occurred, this handset was a Nexus 5 . This testing occurred over one week using the strategy used for the Moto G handset. This showed 
that the performance of the Nexus 5 did not deteriorate over time and offered reliable function. Further to this, this device was tested in a researchers' domicile for over a week and proved to operate reliably-identifying all egress events.

Due to the considerable cost of a high-end Android-based smartphone, such as a Nexus 5, an alternative listener based upon a Raspberry Pi model 3 was evaluated. The evaluation process was as performed with the Nexus 5 and Moto G. During this evaluation process the Raspberry Pi based listener operated reliably over a number of weeks and across approximately 200 simulated egress detections. Following determination of appropriate listener platforms, an evaluation was performed within a lab-based environment, the results of this evaluation is presented in Section 4.2.

\subsection{Lab-Based Evaluation}

Before deployment of this solution to a residential care home, an evaluation of the solution within a simulated, lab-based, environment was performed. In this evaluation the listener was deployed to within a shielded box. This box was placed near a door into an area representing the exterior of a residential care home. The shielding was placed in a manner to focus observation within an egress detection zone. This placement is presented in Figure 7.

Once placed, a series of 187 egress tests were performed over several weeks by researchers entering the area through the door depicted in Figure 7a. These researchers subsequently exited the area via one of two additional doors, once of which is depicted in Figure $7 \mathrm{~b}$. This exiting movement was performed at a range of gait speeds from slow to extremely rapid. During this evaluation the system detected all 187 of the egress events and issued alerts as appropriate, with no false positives. Following this evaluation, the solution was deployed to a residential care home environment where it was tested by researchers on installation and subsequently tested independently by caregivers. This evaluation is presented is Section 4.3 .

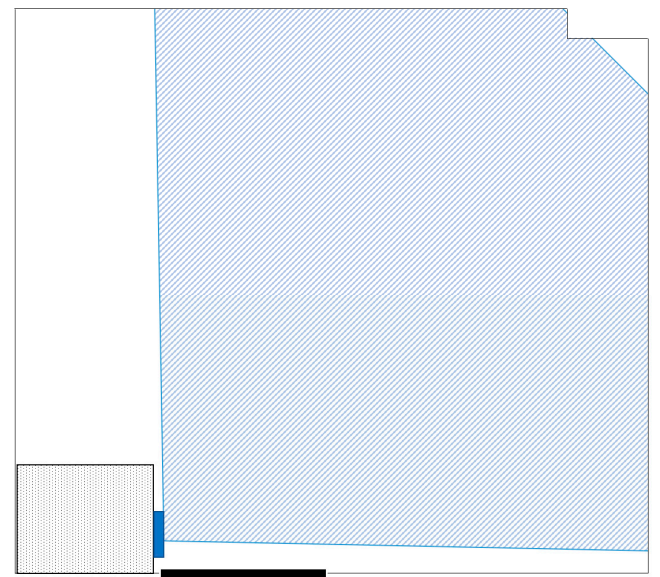

(a)

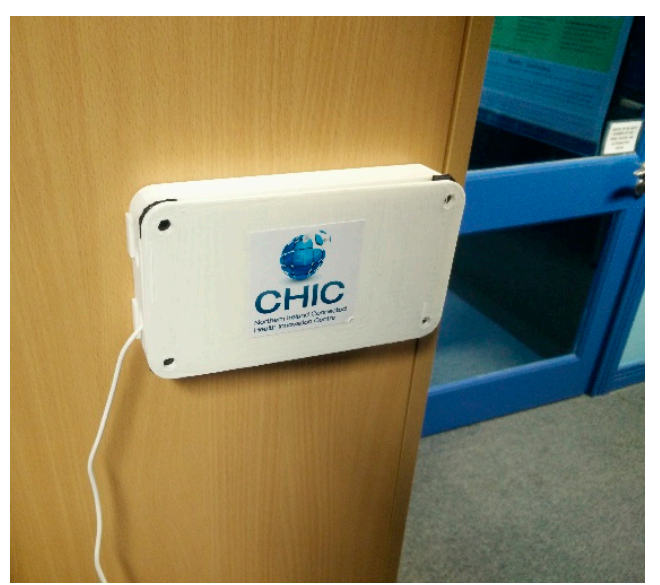

(b)

Figure 7. The deployment of the solution during the lab-based simulation: (a) illustrates a topographical view of the listener placed in context of the environment, the shaded blue area is the egress detection zone, the solid blue box represents the listener and the black square represents the door used to evaluate egress detection; (b) depicts the listener deployed in this test environment.

\subsection{Evaluation within a Residential Care Home}

Following lab-based evaluation, when the researchers were confident about the operation of the solution, it was deployed to a residential care home to monitor unsafe egress of a number of their inhabitants. This deployment is presented in Figure 8.

After installation, the solution was tested by researchers to determine utility. A series of 34 tests were performed where 34 alerts were generated, with no false positives. Caregiving staff within the care home subsequently performed a series of evaluations of the system over several days. In their 
evaluation 54 alerts were generated with no false positive or missed detections. Following this, the solution was used to monitor at risk inhabitants.

Overall the solution proved to operate accurately and detect egress of individuals reliably with no false positives. However, there are some considerations which are elaborated on in Section 5.

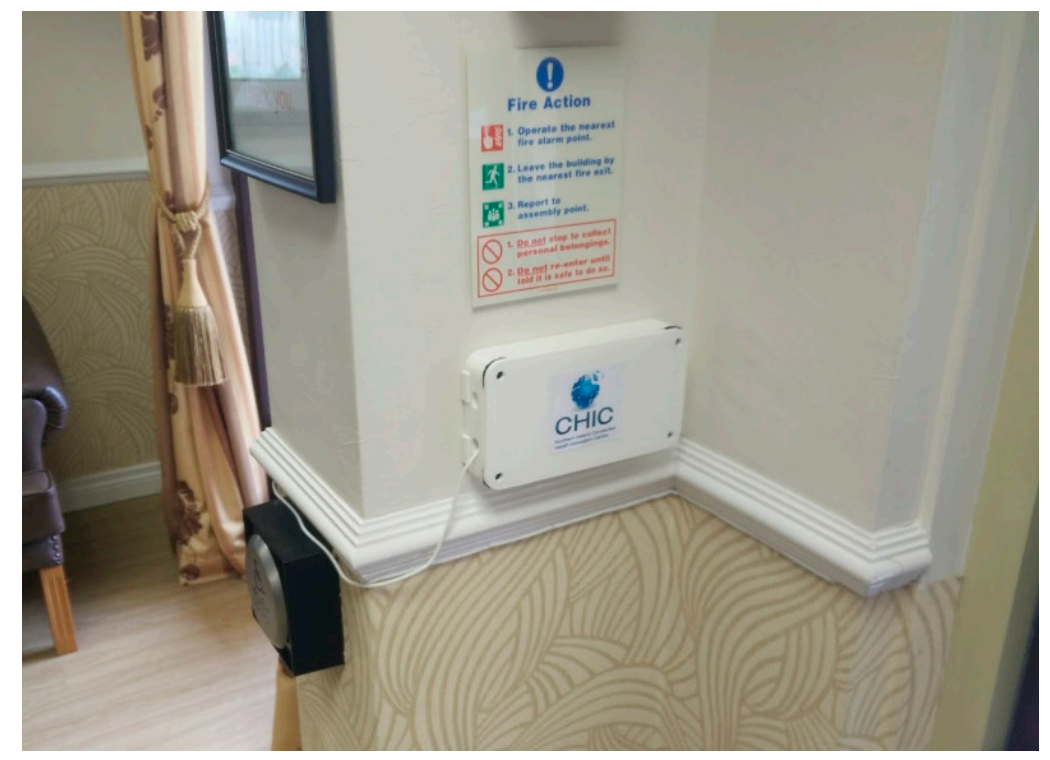

Figure 8. The deployment of the solution within a residential care home. This was subsequently used to monitor unsafe egress of a number of residents.

\section{Concluding Comments and Future Works}

This study has produced a Bluetooth LE based solution which can monitor egress of inhabitants of a residential environment. This solution addresses a number of deficiencies with previous approached to egress monitoring. This egress monitoring provides valuable alerts to caregiving staff, particularly when residents are suffering from aging related cognitive impairments, such as Alzheimer's Disease where interventions may be lifesaving.

The solution was evaluated in three different stages with the final configuration offering reliable and accurate monitoring of such egress events. Notably, 275 egress events were tested using the devised solution with a 100\% detection rate that produced no false positives. These egress events were tested both by researchers and caregiving staff. Following testing, this solution has been deployed to a residential care home where it is being used to monitor unsafe egress of a number of at-risk inhabitants. This solution has been deployed to this care home for over 12 months. Future work may directly incorporate this solution into the fabric of a new residential care home facility being built called Copelands. Other advancements may include integration of modular shielding to better control the egress detection zone or integration of an antennae array and advanced algorithms to provide egress zones to be redefined though modification of software. Finally, use of other positioning technologies such as active RFID tags and Ultra-wideband band markers may be investigated to determine which technology offers the most appropriate operation.

Acknowledgments: Invest Northern Ireland is acknowledged for supporting this project under the Competence Centre Programs Grant RD0513853-Connected Health Innovation Centre.

\section{References}

1. United Nations. Concise Report on the World Population Situation in 2014; United Nations: New York, NY, USA, 2014.

2. United Nations. World Population Prospects: The 2017 Revision; United Nations: New York, NY, USA, 2017.

3. Bakshi, G.S.; Chen, Z. Baby boom, population aging, and capital markets. J. Bus. 1994, 67, 165-202. 
4. Ortman, J.; Velkoff, V.; Hogan, H. An Aging Nation: The Older Population in the United States: Population Estimates and Projection; US Census Bureau Website; US Census Bureau: Suitland, MD, USA, May 2014.

5. Alzheimer's Association. 2017 Alzheimer's disease facts and figures. Alzheimers Dement. 2017, 13, $325-373$.

6. United Nations. World Population Ageing 2009 (Population Studies Series); United Nations: New York, NY, USA, 2010; ISBN 9789211514681.

7. O'Malley, M.; Innes, A.; Wiener, J.M. Decreasing spatial disorientation in care-home settings: How psychology can guide the development of dementia friendly design guidelines. Dementia 2017, 16, 315-328.

8. Zingmark, K. Promoting a good life among people with Alzheimer's disease. J. Adv. Nurs. 2002, 38, 50-58, doi:10.1046/j.1365-2648.2002.02145.x.

9. Peng, L.-M.; Chiu, Y.-C.; Liang, J.; Chang, T.H. Risky wandering behaviors of persons with dementia predict family caregivers' health outcomes. Aging Ment. Health 2017, 1-8, doi:10.1080/13607863.2017.1387764.

10. Reisberg, B.; Borenstein, J.; Salob, S.P.; Ferris, S.H. Behavioral symptoms in Alzheimer's disease: Phenomenology and treatment. J. Clin. Psychiatry 1987, 48, 9-15.

11. Alzheimer's Association. 2018 Alzheimer's Disease facts and figures. Alzheimer's Dement. 2018, 14, $367-429$.

12. Lin, Q.; Zhang, D.; Chen, L.; Ni, H.; Zhou, X. Managing elders' wandering behavior using sensors-based solutions: A survey. Int. J. Gerontol. 2014, 8, 49-55, doi:10.1016/j.ijge.2013.08.007.

13. Rafferty, J.; Synnott, J.; Nugent, C. A Hybrid Rule and Machine Learning Based Generic Alerting Platform for Smart Environments. Engineering in Medicine and Biology Society. In Proceedings of the 2016 38th Annual International Conference of the IEEE Engineering in Medicine and Biology Society (EMBC), Lake Buena Vista, FL, USA, 16-20 August 2016.

14. Helmy, J.; Helmy, A. The Alzimio App for Dementia, Autism Alzheimer's: Using Novel Activity Recognition Algorithms and Geofencing. In Proceedings of the 2016 IEEE International Conference on Smart Computing (SMARTCOMP), St. Louis, MO, USA, 18-20 May 2016; pp. 1-6.

15. Cantón Paterna, V.; Calveras Augé, A.; Paradells Aspas, J.; Pérez Bullones, M.A. A Bluetooth Low Energy Indoor Positioning System with Channel Diversity, Weighted Trilateration and Kalman Filtering. Sensors 2017, 17, 2927.

16. Lin, Y.-J.; Chen, H.-S.; Su, M.-J. A cloud based Bluetooth Low Energy tracking system for dementia patients. In Proceedings of the 2015 Eighth International Conference on Mobile Computing and Ubiquitous Networking (ICMU), Hakodate City, Japan, 20-22 January 2015; pp. 88-89.

17. Issoufaly, T.; Tournoux, P.U. BLEB: Bluetooth Low Energy Botnet for large scale individual tracking. In Proceedings of the 2017 1st International Conference on Next Generation Computing Applications (NextComp), Pointe aux Biches, Mauritius, 19-21 July 2017; pp. 115-120.

18. Mhamdi, J.; Abkari, S. El Contriving an RFID system for Alzheimer patients tracking. In Proceedings of the 2015 Third International Workshop on RFID And Adaptive Wireless Sensor Networks (RAWSN), Agadir, Morocco, 13-15 May 2015; pp. 23-28.

19. López-de-Ipiña, D.; Díaz-de-Sarralde, I.; Zubía, J. An Ambient Assisted Living Platform Integrating RFID Data-on-Tag Care Annotations and Twitter. J. Univ. Comput. Sci. 2010, 16, 1521-1538.

20. Garcia, C.G.; Meana-Llorián, D.; G-Bustelo, B.C.P.; Lovelle, J.M.C.; Garcia-Fernandez, N. Midgar: Detection of people through computer vision in the Internet of Things scenarios to improve the security in Smart Cities, Smart Towns, and Smart Homes. Futur. Gener. Comput. Syst. 2017, 76, 301-313.

21. Wang, J.; Zhang, S.; Shi, R. Vision Based Target Tracking that Distinguishes Facial Feature Targets 2018. U.S. Patent Application No. 15/792,487, 26 April 2018.

22. Ye, M.; Ma, A.J.; Zheng, L.; Li, J.; Yuen, P.C. Dynamic label graph matching for unsupervised video reidentification. In Proceedings of the International Conference on Commuter Vision, Nice, France, 23-27 July 2017.

23. Choudhury, T.; Clarkson, B.; Jebara, T.; Pentland, A. Multimodal person recognition using unconstrained audio and video. In Proceedings of the International Conference on Audio-and Video-Based Person Authentication, Washington, DC, USA, 22-24 March 1999; pp. 176-181.

24. McLaughlin, N.; del Rincon, J.; Miller, P. Recurrent convolutional network for video-based person reidentification. In Proceedings of the IEEE Conference on Computer Vision and Pattern Recognition, Las Vegas, NV, USA, 26 June-1 July 2016; pp. 1325-1334. 
25. BenAbdelkader, C.; Cutler, R.; Davis, L. Stride and cadence as a biometric in automatic person identification and verification. In Proceedings of the Fifth IEEE International Conference on Automatic Face and Gesture Recognition, Washington, DC, USA, 21-21 May 2002; pp. 372-377.

26. Hamdoun, O.; Moutarde, F.; Stanciulescu, B.; Steux, B. Person re-identification in multi-camera system by signature based on interest point descriptors collected on short video sequences. In Proceedings of the Second ACM/IEEE International Conference on Distributed Smart Cameras ICDSC 2008, Stanford, CA, USA, 7-11 September 2008; pp. 1-6.

27. Saranya, S.; JesuJayarin, P. A survey for tracking and monitoring the alzheimer patient. In Proceedings of the 2017 Third International Conference on Science Technology Engineering \& Management (ICONSTEM), Tamil Nadu, India, 23-24 March 2017; pp. 116-120.

28. Shenvi, P.; Baheria, P.; Jose, S.; Kumar, S.; Nayak, J.S. Wearable Tracking Device for Alzheimer's Patients: A Survey 2016. JETIR 2016, 3, 147-151.

29. Garzon, S.R.; Arbuzin, D.; Küpper, A. Geofence Index: A Performance Estimator for the Reliability of Proactive Location-Based Services. In Proceedings of the 2017 18th IEEE International Conference on Mobile Data Management (MDM), Daejeon, Korea, 29 May-1 June 2017; pp. 1-10.

30. Mendoza, M.B.; Bergado, C.A.; Castro, J.L.B. De; Siasat, R.G.T. Tracking system for patients with Alzheimer's disease in a nursing home. In Proceedings of the TENCON 2017-2017 IEEE Region 10 Conference, Penang, Malaysia, 5-8 November 2017; pp. 2566-2570.

31. Shree, S.R.B.; Sheshadri, H.S.; Shivakumar, R.; Kumar, H.S.V. Design of embedded system for tracking and locating the patient suffering from Alzheimer's disease. In Proceedings of the 2014 IEEE International Conference on Computational Intelligence and Computing Research, Tamilnadu, India, 18-20 December 2014; pp. 1-5.

32. Clark, B.K.; Winkler, E.A.; Brakenridge, C.L.; Trost, S.G.; Healy, G.N. Using Bluetooth proximity sensing to determine where office workers spend time at work. PLOS ONE 2018, 13, e0193971.

33. Deng, D.; Yuan, H.; Lv, J.; Ju, Y. WSN-Based Space Charge Density Measurement System. PLoS ONE 2017, 12, e0169034.

34. Rafferty, J.; Synnott, J.; Ennis, A.; Nugent, C.; McChesney, I.; Cleland, I. SensorCentral: A Research Oriented, Device Agnostic, Sensor Data Platform; Springer: Cham, Switzerland, 2017; Volume 10586, ISBN 9783319675848 .

35. Google Firebase Cloud Messaging. Available online: https:/firebase.google.com/docs/cloud-messaging/ (accessed on 1 April 2018).

36. Gao, V. Proximity and RSSI. Available online: http://blog.bluetooth.com/proximity-and-rssi (accessed on 01 April 2018).

37. Feng, X.; Shen, J.; Fan, Y. REST: An alternative to RPC for Web services architecture. In Proceedings of the First International Conference on Future Information Networks, ICFIN 2009, Beijing, China, 14-17 October 2009; pp. 7-10.

38. Rafferty, J.; Synnott, J.; Nugent, C.; Ennis, A.; Catherwood, P.; McChesney, I.; Cleland, I. A Scalable, Research Oriented, Generic, Sensor Data Platform. IEEE Access 2018, 6, 45473-45484.

39. Gupta, A.; Gaffar, A. Hybrid Application Development using Ionic Framework \& AngularJS. Int. J. Innov. Res. Comput. Sci. Technol. 2016, 4, 62-64.

(C) 2018 by the authors. Licensee MDPI, Basel, Switzerland. This article is an open access article distributed under the terms and conditions of the Creative Commons Attribution (CC BY) license (http://creativecommons.org/licenses/by/4.0/). 